\title{
Sharp estimates for the hitting probability on time-dependent barriers for a Brownian Motion. Weak approximation of a Brownian motion killed on time-dependent barriers
}

\author{
Lucia Caramellino ${ }^{1}$ and Barbara Pacchiarotti ${ }^{2}$ \\ ${ }^{1}$ Dipartimento di Matematica, \\ Università di Roma Tor Vergata, via della Ricerca Scientifica, I-00133 Roma, Italy; \\ Email: caramell@mat.uniroma2.it fax: + 390672594699 \\ 2 Dipartimento di Matematica, \\ Università di Roma Tor Vergata, via della Ricerca \\ Scientifica, I-00133 Roma, Italy \\ Email: pacchiar@mat.uniroma2.it; fax: + 390672594699
}

\begin{abstract}
We deal with the problem of the numerical evaluation by Monte Carlo methods of the expectation of a function of the position at a fixed time of a Brownian motion killed when it reaches time-dependent barriers, a problem of interest in Finance, e.g. for the pricing of barrier options. Two stochastic Euler schemes are compared in this paper: the "discrete" scheme and the "continuous" one. The latter has been already introduced in literature and makes use of some sharp large deviation estimates of the exit probability for a Brownian bridge in a small time interval. We show that, in the weak sense, the "continuous" scheme is much faster than the "discrete" one, by proving an inequality which provides a comparison between the rates of convergence.
\end{abstract}

\section{Introduction}

Let $W_{t}$ denote a one-dimensional Brownian Motion starting at $x$, with constant drift $\mu$ and constant diffusion coefficient $\sigma>0$, that is

$$
W_{t}=x+\mu t+\sigma B_{t}
$$

where $B$ stands for a standard one-dimensional Brownian Motion. Consider two real functions

$$
L, U:[0,+\infty) \rightarrow \mathbb{R}
$$

such that $L(t)<U(t)$, for any $t \geq 0$. The maps $L$ and $U$ play the role of the lower and upper barrier, respectively. In this paper we study approximations of expectations of the type

$$
\mathbb{E}\left[f\left(W_{T}\right) \mathbb{1}_{\{\tau>T\}}\right],
$$

or also $\mathbb{E}\left[f\left(W_{T}\right) \mathbb{1}_{\{\tau \leq T\}}\right]$, where $f$ is a nonnegative Borel measurable function and $\tau$ is the first hitting time on the barriers, that is

$$
\tau=\inf \left\{t>0: W_{t} \leq L(t) \text { or } W_{t} \geq U(t)\right\} .
$$


The problem of the (numerical) evaluation of expectations as in (2) is quite interesting and arises naturally in a financial framework to the pricing of barrier options. Barrier options differ from the well-known European conventional options by means of the introduction of one or two boundaries, deterministic and time-dependent, which are contractually specified and which may nullify the value of the option if breached by the underlying asset price. Such options are increasingly popular in the financial markets because they are less expensive than conventional options. For instance a knock-out barrier call is equivalent to the corresponding standard call provided that the underlying asset price does not hit either barrier, otherwise its payoff is set equal to zero. Under the Black and Scholes model for the underlying asset price, the pricing formula of a knock-out call is

$$
e^{-r T} \mathbb{E}\left[\max \left(e^{W_{T}}-K, 0\right) \mathbb{1}_{\tau>T}\right],
$$

where $K$ denotes the exercise price, $T$ is the maturity and in (1), as usual, $\sigma$ stands for the volatility and $\mu=r-\sigma^{2} / 2, r$ being the (constant) spot rate. In order to compute the price of barrier options, one needs to know the law of $W_{T}$ killed at time $\tau$. This is possible only in the case of constant or linear barriers, see e.g. Revuz and Yor [10] for the formula of the exit probability and Kunitomo and Ikeda [9] for the pricing formula for such kind of barriers. Otherwise, this law is not explicitly known and some other numerical methods have to be used. For example, Geman and Yor [7] developed a Laplace transform approach and Boyle and Tian [4] introduced a numerical method involving trinomial models. Moreover, in the case of a single barrier, it is immediate to show that the function $(s, x) \mapsto v(s, x) \stackrel{\text { def }}{=} \mathbb{E}_{s, x}\left[f\left(W_{T}\right) \mathbb{I}_{\tau>T}\right]$ solves the parabolic differential problem

$$
\begin{array}{ll}
\frac{\partial}{\partial s} v(s, x)+L_{s} v(s, x)=0 & \text { for }(s, x) \in[0, T) \times[0,+\infty) \\
v(s, x)=0 & \text { for }(s, x) \in[0, T) \times(-\infty, 0) \\
v(T, x)=f(x+g(T)) & \text { for } x \in(0,+\infty)
\end{array}
$$

where

$$
L_{s}=\left(\left(r-\frac{1}{2} \sigma^{2}\right) s-g^{\prime}(s)\right) \frac{\partial}{\partial x}+\frac{1}{2} \frac{\partial^{2}}{\partial x^{2}}
$$

and the function $g$ stands for the barrier. Thus, one could numerically solve problem (5) in order to numerically compute $v(s, x)$, that is the price of a single knock-out barrier option.

Furthermore, the numerical valuation of the price can be done via Monte Carlo algorithms, as in Baldi, Caramellino and Iovino [2], [3] and, in a multidimensional setting, in Gobet [8]. In this context, the process $W$ is simulated at fixed times $t_{j}=j T / n$, with $n$ large enough, and typically the exit time $\tau$ is approximated by means of the first instant $t_{j}$ such that $W_{t_{j}}$ is outside the barriers, if it does exist (if $W$ is a more general diffusion process and it is not possible to simulate exactly $W$, also $W$ is approximated by means of some discretization scheme, e.g. the Euler scheme). Let $\tau_{n}^{d}$ denote the approximating hitting time. One then collects a large number $\tau_{n}^{d,(m)}$ and $W_{T}^{(m)}$ of independent approximating exit times and positions of the process at the maturity and finally approximates the expectation by means of the empirical mean of the observed payoffs:

$$
\mathbb{E}\left[f\left(W_{T}\right) \mathbb{I}_{\{\tau \leq T\}}\right] \simeq \mathbb{E}\left[f\left(W_{T}\right) \mathbb{1}_{\left\{\tau_{n}^{d} \leq T\right\}}\right] \simeq \frac{1}{M} \sum_{m=1}^{M} f\left(W_{T}^{(m)}\right) \mathbb{I}_{\left\{\tau_{n}^{d,(m)}>T\right\}}
$$


This kind of procedure gives rise to two errors: the first depending on the choice of the number $n$ giving the discretization step $T / n$ and the second coming from the number $M$ of independent simulations. Concerning the former, that is the one we are interested in, the above procedure could be considered exact in the case of discrete monitoring (see e.g. Broady, Galssermann and Kou [5]) but since the barriers are supposed to be monitored continuously in time, the procedure works very poorly and the error goes to 0 as $n \rightarrow \infty$ very slowly, as it has been pointed out by many authors (see for instance [2] and references quoted therein). In order to improve the speed of convergence to 0 for such an error, in [2] a sharp large deviation estimate of the conditional probability that the barriers are reached during $\left[t_{j}, t_{j+1}\right]$ by the process $W$, given its observations at times $t_{j}$ and $t_{j+1}$, is computed and used in order to kill the process. This gives another approximation $\tau_{n}^{c}$ of the exit time $\tau$ and empirical studies show that the approximation $\tau_{n}^{c}$ works much more better than $\tau_{n}^{d}$ : setting $\mathcal{E}_{n}^{c}(f)=\left|\mathbb{E}\left[f\left(W_{T}\right) \mathbb{I}_{\{\tau>T\}}\right]-\mathbb{E}\left[f\left(W_{T}\right) \mathbb{I}_{\left\{\tau_{n}^{c}>T\right\}}\right]\right|$ and $\mathcal{E}_{n}^{d}(f)=\left|\mathbb{E}\left[f\left(W_{T}\right) \mathbb{1}_{\{\tau>T\}}\right]-\mathbb{E}\left[f\left(W_{T}\right) \mathbb{1}_{\left\{\tau_{n}^{d}>T\right\}}\right]\right|$, then

$$
\lim _{n \rightarrow \infty} \frac{\mathcal{E}_{n}^{c}(f)}{\mathcal{E}_{n}^{d}(f)}=0 .
$$

In this brief paper, we quantify how much the second procedure improves the first one by showing that

$$
\frac{\mathcal{E}_{n}^{c}(f)}{\mathcal{E}_{n}^{d}(f)} \leq \frac{c_{n}}{n}+\bar{\gamma}_{n}
$$

where $c_{n}$ is a bounded positive sequence and $\bar{\gamma}_{n}=o\left(1 / n^{\beta}\right)$ for any $\beta>0$. Moreover, in the double constant or linear barrier case it holds $c_{n}=0$ (if a single constant or linear barrier is taken into account then $\mathcal{E}_{n}^{c}(f)=0$ because the exit probability used in the discretization turns out to be exact, as remarked in [2]).

Although the process $W$ here considered is merely a Brownian motion with constant drift, there are not so many results in literature giving the speed of convergence of the errors $\mathcal{E}_{n}^{c}(f)$ and $\mathcal{E}_{n}^{d}(f)$ for general functions $f$. For example, the special case $f(x)=$ $\mathbb{1}_{\{x \in I\}}$, that is $\mathbb{E}\left[f\left(W_{T}\right) \mathbb{1}_{\{\tau>T\}}\right]=P\left(W_{T} \in I, \tau>T\right)$, has been studied by Siegmund and Yuh [11]. They proved that

$$
\mathcal{E}_{n}^{d}(f)=\frac{C}{\sqrt{n}}+o(1 / \sqrt{n})
$$

but only under the constraints that $\mu=0$, the barriers $L$ and $U$ are constant and that $I$ is an interval strictly included in $(L, U)$. Notice that in particular this implies that $f$ vanishes in a neighborhood of the barriers. Such a condition is practically required also by Gobet in [8]. In that paper, the errors $\mathcal{E}_{n}^{c}(f)$ and $\mathcal{E}_{n}^{d}(f)$ are both analyzed, also for general diffusion taking values in a multidimensional setting and the following results are proved:

$$
\mathcal{E}_{n}^{c}(f)=\frac{C}{n}+o(1 / n) \text { and } \mathcal{E}_{n}^{d}(f)=O(1 / \sqrt{n}) .
$$

In the simple case of a Brownian motion with constant barriers or with a single timedependent barrier, these results can be refined in such a way that (6) holds. Nevertheless, in the general case (6) does not follow from the results in [8] since it is not possible to see the double time-dependent barrier case as a particular case of that study. Moreover, it 
worth stressing that (6) will hold for positive functions (with mild integrability conditions) not necessarily zero on the barriers, a constraint which seems to be crucial to use PDE techniques, turning out from (5), allowing to achieve the rate of convergence to 0 of the errors, as done in [8]. Let us finally point out that in a financial framework the vanishing conditions on $f$ are actually restrictive. Indeed, in the cited barrier call option example whose price is given by (4), this implies that the barriers $L$ and $U$ have to be below $\ln K$, that is the logarithm of the exercise price, and this is not the case in many situations. It turns out of interest what happens when the option payoff is strictly positive on the barriers.

The paper is organized as follows. Section 2 is devoted to set up the discretization schemes and to state the result, whose proofs can be found in Section 3.

\section{The result}

Let us first recall how the numerical approximation introduced in [2] works.

Let $t_{0}=0<t_{1}<\cdots<t_{n}=T$ be a partition of the time interval $[0, T]$ such that $t_{j+1}-t_{j}=T / n, j=0,1, \ldots, n-1$. At each step, the value $W_{t_{j}}$ is simulated and the "discrete" procedure sets the hitting time $\tau_{n}^{d}$ equal to the first instant $t_{j}$ in which $W_{t_{j}}$ crosses a boundary. $\tau_{n}^{d}$ provides an overestimate of the (continuously monitored) hitting time $\tau$ since $W_{t_{j}}$ and $W_{t_{j+1}}$ might not have breached the barriers while $W_{t}$ had for some $t \in\left(t_{j}, t_{j+1}\right)$. To account for this, one could think to use the probability $p_{n}^{j}$ that $W_{t_{j}}$ hits on the barriers during the time interval $\left(t_{j}, t_{j+1}\right)$, given the observations $W_{t_{j}}$ and $W_{t_{j+1}}$. Thus one can with probability $p_{n}^{j}$ stop the simulation and set the approximating exit time $\tau_{n}^{c}$ of this "continuous" monitoring equal to $t_{j}$.

If $p_{n}^{j}$ was known, this procedure would provide a quite precise result, whose bias would be the smallest one and being actually exact whenever one is interested in the approximation of the hitting time through its distribution function. Unfortunately, the probability $p_{n}^{j}$ can be exactly computed only in some very special cases, for instance, for constant or linear barriers (in the double barrier case, the formula of the exit probability from two linear barriers is known in infinite series form, see [10], pp. 105-106). As introduced in [1] for fixed domains and in [2] for time-dependent barriers, one could use a sharp estimate $\hat{p}_{n}^{j}$ for $p_{n}^{j}$ allowing to set up the simulation procedure as described above. Indeed, by suitably developing some sharp large deviation results firstly proved by Fleming and James in [6], the following result holds:

Theorem 2.1 Suppose that $L$ and $U$ are continuous with Lipschitz continuous derivatives and let us set $L_{j}=L\left(t_{j}\right)$ and $U_{j}=U\left(t_{j}\right)$. Then for every $(x, y) \in\left(L_{j}, U_{j}\right) \times\left(L_{j+1}, U_{j+1}\right)$,

$$
p_{n}^{j}(x, y)=\widehat{p}_{n}^{j}(x, y)\left(1+\mathcal{R}_{j, n}(x, y)\right)
$$

where

$$
\hat{p}_{n}^{j}(x, y)=\kappa \cdot \exp \left(-\frac{2 n}{\sigma^{2}} \min \left(\left(L_{j}-x\right)\left(L_{j+1}-y\right),\left(U_{j}-x\right)\left(U_{j+1}-y\right)\right)\right)
$$

being

$$
\kappa= \begin{cases}1 & \text { if }\left(U_{j}-x\right)\left(U_{j+1}-y\right) \neq\left(L_{j}-x\right)\left(L_{j+1}-y\right) \\ 2 & \text { if }\left(U_{j}-x\right)\left(U_{j+1}-y\right)=\left(L_{j}-x\right)\left(L_{j+1}-y\right),\end{cases}
$$

and $\mathcal{R}_{j, n}(x, y)$ goes to 0 as $n \rightarrow \infty$ as or faster than $\frac{1}{n}$. 
Remark 2.2 The above result is actually a little modification of what proved in [2], Theorem 2.1 and Corollary 2.2. Indeed, suppose for instance that $\left(U_{j}-x\right)\left(U_{j+1}-y\right)<$ $\left(L_{j}-x\right)\left(L_{j+1}-y\right)$, so that for $n$ large enough we can suppose that also $\left(U_{j}-x\right)\left(U_{j}-y\right)<$ $\left(L_{j}-x\right)\left(L_{j}-y\right)$ holds and thus $x+y>U_{j}+L_{j}$. Then Theorem 2.1 in [2] gives

$$
p_{n}^{j}(x, y)=\exp \left(-\frac{2 n}{\sigma^{2}}\left(U_{j}-x\right)\left(U_{j}-y\right)-\frac{2}{\sigma^{2}}\left(U_{j}-x\right) U_{j}^{\prime}\right)(1+O(1 / n))
$$

where $U_{j}^{\prime}=U^{\prime}\left(t_{j}\right)$. Now, since $U$ has a Lipschitz continuous derivative, we can write $U_{j}+U_{j}^{\prime} / n=U_{j+1}+O\left(1 / n^{2}\right)$, so that

$$
\begin{aligned}
& -\frac{2 n}{\sigma^{2}}\left(U_{j}-x\right)\left(U_{j}-y\right)-\frac{2}{\sigma^{2}}\left(U_{j}-x\right) U_{j}^{\prime}=-\frac{2 n}{\sigma^{2}}\left(U_{j}-x\right)\left(U_{j}+\frac{1}{n} U_{j}^{\prime}-y\right) \\
= & -\frac{2 n}{\sigma^{2}}\left(U_{j}-x\right)\left(U_{j+1}-y+O\left(1 / n^{2}\right)\right)=-\frac{2 n}{\sigma^{2}}\left(U_{j}-x\right)\left(U_{j+1}-y\right)+O(1 / n)
\end{aligned}
$$

and

$p_{n}^{j}(x, y)=\exp \left(-\frac{2 n}{\sigma^{2}}\left(U_{j}-x\right)\left(U_{j+1}-y\right)+O(1 / n)\right)(1+O(1 / n))=\hat{p}_{n}^{j}(x, y)(1+O(1 / n))$,

$\widehat{p}_{n}^{j}(x, y)$ being defined in (8). Similar arguments apply if $\left(U_{j}-x\right)\left(U_{j+1}-y\right) \geq\left(L_{j}-\right.$ $x)\left(L_{j+1}-y\right)$.

In conclusion, we can set the approximating exit times as follows:

$$
\begin{aligned}
\tau_{n}^{c}=\inf \left\{t_{j}: \mathbb{1}_{\left[0, \widehat{p}_{j}^{n}\right]}\left(Z_{j}^{n}\right)=1\right\} & \text { as for the "continuous" case } \\
\tau_{n}^{d}=\inf \left\{t_{j}: W_{t_{j}} \leq L\left(t_{j}\right) \text { or } W_{t_{j}} \geq U\left(t_{j}\right)\right\} & \text { as for the "discrete" case }
\end{aligned}
$$

where $\left\{Z_{j}^{n}\right\}_{\{j=1, \ldots, n ; n \geq 1\}}$ is a sequence of i.i.d. uniformly distributed on $[0,1]$ r.v.'s independent of $W$.

Take now $f: \mathbb{R} \rightarrow \mathbb{R}$ a Borel measurable function. Let us define

$$
\begin{array}{ll}
\mathcal{E}_{n}^{c}(f)=\left|\mathbb{E}\left[f\left(W_{T}\right) \mathbb{I}_{\{\tau>T\}}\right]-\mathbb{E}\left[f\left(W_{T}\right) \mathbb{I}_{\left\{\tau_{n}^{c}>T\right\}}\right]\right| & \text { as the "continuous" error } \\
\mathcal{E}_{n}^{d}(f)=\left|\mathbb{E}\left[f\left(W_{T}\right) \mathbb{1}_{\{\tau>T\}}\right]-\mathbb{E}\left[f\left(W_{T}\right) \mathbb{I}_{\left\{\tau_{n}^{d}>T\right\}}\right]\right| & \text { as the "discrete" error }
\end{array}
$$

whenever the above expectations exist. In papers [2] and [3] (see also references quoted therein), numerical results empirically show that $\mathcal{E}_{n}^{c}(f)$ goes to 0 faster than $\mathcal{E}_{n}^{c}(f)$. Here, we compare these two errors by showing how fast un upper bound for their ratio has to converge to 0 as $n \rightarrow \infty$ :

Theorem 2.3 Suppose that the barriers $L$ and $U$ are $\mathcal{C}^{1}$ functions with Lipschitz continuous derivatives. Let $f$ denote a positive function such that $\mathbb{E}\left[f^{1+\epsilon}\left(W_{T}\right)\right]$ exists for some $\epsilon>0$. Then, for any $\alpha>0$, it holds

$$
\mathcal{E}_{n}^{c}(f) \leq \mathcal{E}_{n}^{d}(f)\left(\frac{c_{n}}{n}+\frac{d_{n}}{n^{\alpha}}\right)+\gamma_{n}
$$

where 
- $c_{n}=\frac{2 M K^{\prime}}{\sigma^{2}} e^{\frac{2 M K^{\prime}}{n \sigma^{2}}}$ and $d_{n}=e^{\frac{4 M K^{\prime}}{n \sigma^{2}}}$, being $M=\max _{t \in[0, T]}(U(t)-L(t))$ and $K^{\prime}=$ $\max \left(K_{L}^{\prime}, K_{U}^{\prime}\right), K_{L}^{\prime}$ and $K_{U}^{\prime}$ denoting the Lipschitz constant of $L^{\prime}$ and $U^{\prime}$, respectively;

- $\left\{\gamma_{n}\right\}_{n}$ is a positive sequence going to 0 as $n \rightarrow \infty$ faster than any power of $\frac{1}{n}$ (i.e. $\lim _{n \rightarrow \infty} n^{k} \gamma_{n}=0$, for any $k$ ).

Theorem 2.3 then ensures that the continuous scheme is faster than the discrete one, whose speed of convergence $\mathcal{E}_{n}^{d}(f)$ is known only for some particular cases for $f$, as already mentioned in the Introduction. Some conjectures assert that $\mathcal{E}_{n}^{d}(f)=O(1 / \sqrt{n})$ for any $f$. Theorem 2.3 claims that the corrected error $\mathcal{E}_{n}^{c}(f)$ has to go to 0 , as $n \rightarrow \infty$, faster than $\mathcal{E}_{n}^{d}(f)$ and also $\mathcal{E}_{n}^{c}(f)=o(1 / n)$. Moreover, in the constant or linear barrier case, one has $c_{n}=0$ because $K^{\prime}=0$, so that the convergence is actually very fast, almost of exponential-type, since it is the same as $\frac{1}{n^{\alpha}}$ for any $\alpha>0$.

Theorem 2.3 will be proved in the next section. It is based on a result (Proposition 3.3) through which we can state also similar results when the function $f$ is not positive and for more general expectations allowing to handle barrier options with rebate. For details, see Remark 3.4 and Remark 3.5.

\section{Proofs}

The proof of Theorem 2.3 needs several preliminary results so that it is postponed to the end of this section.

Let us firstly introduce the notations we are going to use.

- $L_{j}=L\left(t_{j}\right)$ and $U_{j}=U\left(t_{j}\right)$, where $t_{j}=j T / n$, for $j=0 \ldots n$.

- $\mu_{n}$ stands for the joint law of $W_{t_{1}}, \ldots, W_{t_{n}}$ :

$$
\mu_{n}(d x)=P\left(W_{t_{1}} \in d x_{1}, \ldots W_{t_{n}} \in d x_{n}\right) .
$$

- $p_{n}^{j}(x, y)$ is the conditional exit probability of $W$ during the time interval $\left[t_{j}, t_{j+1}\right]$, given the positions $x$ and $y$ of the process $W$ at times $t_{j}$ and $t_{j+1}$, respectively.

- $\widehat{p}_{n}^{j}(x, y)$ stands for the asymptotics of $p_{n}^{j}(x, y)$ given by Theorem 2.3 (see (8)).

- $q_{n}(x)$ denotes the exit probability conditional to the positions $x=\left(x_{1}, \ldots, x_{n}\right)$ of $W$ at times $\left(t_{1}, \ldots, t_{n}\right)$ :

$$
q_{n}(x)=1-\prod_{j=0}^{n-1}\left(1-p_{n}^{j}\left(x_{j}, x_{j+1}\right)\right) .
$$

Since the conditional laws of $\left(W_{t}\right)_{t \in\left[t_{j}, t_{j+1}\right]}$ given the position $x_{j}$ and $x_{j+1}$ at times $t_{j}$ and $t_{j+1}$, respectively turn out to be independent of each other, the above representation for $q_{n}$ actually holds. Notice that $p_{n}^{j}(x, y)$ is the probability of hitting on the barriers for a Brownian bridge, starting at $x$ at time $t_{j}$ and pinned by $y$ at time $t_{j+1}$. 
- $\widehat{q}_{n}(x)$ denotes the approximation of $q_{n}(x)$ turning out by considering the approximations $\hat{p}_{n}^{j}$ of $p_{n}^{j}$ :

$$
\widehat{q}_{n}(x)=1-\prod_{j=0}^{n-1}\left(1-\hat{p}_{n}^{j}\left(x_{j}, x_{j+1}\right)\right) .
$$

In order to study the distance between $q_{n}$ and $\widehat{q}_{n}$ in terms of the distance among the $p_{n}^{j}$ 's and the $\widehat{p}_{n}^{j}$ 's, which will be done in the sequel, we need the following simple lemma:

Lemma 3.1 For a fixed integer $m$, let us define

$$
\rho_{m}(p)=1-\prod_{k=1}^{m}\left(1-p_{k}\right)
$$

where $p=\left\{p_{k}\right\}_{k} \subset[0,1]$. Let $\gamma=\left\{\gamma_{k}\right\}_{k}$ and $\eta=\left\{\eta_{k}\right\}_{k}$ be sequences in $[0,1]$ such that, for some constant $\beta>0$,

$$
\left|\gamma_{k}-\eta_{k}\right| \leq \beta \gamma_{k} \quad \text { for any } k \geq 1
$$

Then, for any integer $n$,

$$
\left|\rho_{n}(\gamma)-\rho_{n}(\eta)\right| \leq \beta \rho_{n}(\gamma) .
$$

Proof. We prove the statement by induction on $n$. If $n=1$, the statement easily follows. Suppose now it holds for $n$. One can write $\rho_{n+1}(p)=p_{n+1}+\left(1-p_{n+1}\right) \rho_{n}(p)$, so that

$$
\begin{gathered}
\rho_{n+1}(\gamma)-\rho_{n+1}(\eta)=\left(\gamma_{n+1}-\eta_{n+1}\right)+\left(1-\gamma_{n+1}\right) \rho_{n}(\gamma)-\left(1-\eta_{n+1}\right) \rho_{n}(\eta) \\
=\left(\gamma_{n+1}-\eta_{n+1}\right)\left(1-\rho_{n+1}(\eta)\right)+\left(1-\gamma_{n+1}\right)\left(\rho_{n}(\gamma)-\rho_{n}(\eta)\right)
\end{gathered}
$$

Since $\left|\gamma_{n+1}-\eta_{n+1}\right| \leq \beta \gamma_{n+1}$ and, by induction, $\left|\rho_{n}(\gamma)-\rho_{n}(\eta)\right| \leq \beta \rho_{n}(\gamma)$, one obtains

$$
\left|\rho_{n+1}(\gamma)-\rho_{n+1}(\eta)\right| \leq \beta \gamma_{n+1}+\beta\left(1-\gamma_{n+1}\right) \rho_{n}(\gamma)=\beta \rho_{n+1}(\gamma)
$$

Thus, the statement holds for any $n$.

In the following lemma, we study the distance between $p_{n}^{j}(x, y)$ and $\widetilde{p}_{n}^{j}(x, y)$ that is, we study the asymptotic behavior of the quantity $\mathcal{R}_{j, n}(x, y)$ given by Theorem 2.1 .

Lemma 3.2 For $(x, y) \in\left(L_{j}, U_{j}\right) \times\left(L_{j+1}, U_{j+1}\right)$, let us define

$$
w^{j}(x, y)=\left(U_{j}-x\right)\left(U_{j+1}-y\right)-\left(L_{j}-x\right)\left(L_{j+1}-y\right) \text {. }
$$

Then, there exists a positive integer $n_{0}$ such that for any $\alpha \geq 1, n \geq n_{0}$ and for any $(x, y) \in\left(L_{j}, U_{j}\right) \times\left(L_{j+1}, U_{j+1}\right)$ such that

$$
\left|w^{j}(x, y)\right| \geq \frac{\alpha \sigma^{2} \ln n}{2 n}
$$

one has

$$
\left|p_{n}^{j}(x, y)-\hat{p}_{n}^{j}(x, y)\right| \leq\left(\frac{c_{n}}{n}+\frac{d_{n}}{n^{\alpha}}\right) p_{n}^{j}(x, y)
$$

where

$$
c_{n}=\frac{2 M K^{\prime}}{\sigma^{2}} e^{\frac{2 M K^{\prime}}{n \sigma^{2}}} \quad \text { and } \quad d_{n}=e^{\frac{4 M K^{\prime}}{n \sigma^{2}}}
$$

being $M=\max _{t \in[0, T]}(U(t)-L(t))$ and $K^{\prime}=\max \left(K_{L}^{\prime}, K_{U}^{\prime}\right)$, where $K_{L}^{\prime}$ and $K_{U}^{\prime}$ stand for the Lipschitz constants of $L^{\prime}$ and $U^{\prime}$, respectively. Notice that if both $U$ and $L$ are constant or linear, then $c_{n}=0$ and $d_{n}=1$, for any $n$. 
It is worth remarking that condition (9) follows from the fact that the approximation of the exit probability as given by Theorem 2.1 is not uniform as $(x, y) \in\left(L_{j}, U_{j}\right) \times$ $\left(L_{j+1}, U_{j+1}\right)$, in the sense that it is mainly dependent on the lower barrier if $w_{n}^{j}>0$ and on the upper one whenever $w_{n}^{j}<0$.

Proof. First of all, notice that the asymptotics $\tilde{p}_{n}^{j}$ of $p_{n}^{j}$ turn out to be equal to the asymptotics of the probability of hitting on the two lines joining $\left(t_{j}, L_{j}\right)$ to $\left(t_{j+1}, L_{j+1}\right)$ and $\left(t_{j}, U_{j}\right)$ to $\left(t_{j+1}, U_{j+1}\right)$, respectively. Let us denote these two linear barrier as

$$
\phi_{L}^{j}(t)=n\left(L_{j+1}-L_{j}\right) \cdot\left(t-t_{j}\right)+L_{j} \text { and } \phi_{U}^{j}(t)=n\left(U_{j+1}-U_{j}\right) \cdot\left(t-t_{j}\right)+U_{j} .
$$

The idea of the proof is to construct, for each barrier $L$ and $U$, two further straight lines, i.e. linear barriers, such that for any $t \in\left[t_{j}, t_{j+1}\right]$ then the barrier of interest can be lower and upper bounded by these new linear barriers. This will turn out to be crucial because for linear barriers the formula for the exit probability is known exactly.

Thus, among the straight lines crossing the point $\left(t_{j}, L_{j}\right)$, we consider two straight lines $\phi_{L, 1}^{j}$ and $\phi_{L, 2}^{j}$ which contain the barriers $L(t)$ and $\phi_{L}^{j}(t)$ as $t \in\left[t_{j}, t_{j+1}\right)$ :

$$
\phi_{L, 1}^{j}(t)=L^{\prime}\left(t_{1}^{*}\right) \cdot\left(t-t_{j}\right)+L_{j}, \quad \phi_{L, 2}^{j}(t)=L^{\prime}\left(t_{2}^{*}\right) \cdot\left(t-t_{j}\right)+L_{j}
$$

where $t_{1}^{*}, t_{2}^{*} \in\left[t_{j}, t_{j+1}\right]$ are defined as

$$
L^{\prime}\left(t_{1}^{*}\right)=\max _{t \in\left[t_{j}, t_{j+1}\right]} L^{\prime}(t), \quad L^{\prime}\left(t_{2}^{*}\right)=\min _{t \in\left[t_{j}, t_{j+1}\right]} L^{\prime}(t)
$$

Then obviously both $L(t)$ and $\phi_{L}^{j}(t)$ belong to $\left[\phi_{L, 2}^{j}(t), \phi_{L, 1}^{j}(t)\right]$, as $t \in\left[t_{j}, t_{j+1}\right]$. We similarly define $\phi_{U, 1}^{j}$ and $\phi_{U, 2}^{j}$ :

$$
\phi_{U, 1}^{j}(t)=U^{\prime}\left(t_{1}^{\circ}\right) \cdot\left(t-t_{j}\right)+U_{j}, \quad \phi_{U, 2}^{j}(t)=U^{\prime}\left(t_{2}^{\circ}\right) \cdot\left(t-t_{j}\right)+U_{j}
$$

where $t_{1}^{\circ}, t_{2}^{\circ} \in\left[t_{j}, t_{j+1}\right]$ are such that

$$
U^{\prime}\left(t_{1}^{\circ}\right)=\min _{t \in\left[t_{j}, t_{j+1}\right]} U^{\prime}(t), \quad U^{\prime}\left(t_{2}^{\circ}\right)=\max _{t \in\left[t_{j}, t_{j+1}\right]} U^{\prime}(t) .
$$

Therefore, $U(t), \phi_{U}^{j}(t) \in\left[\phi_{U, 1}^{j}(t), \phi_{U, 2}^{j}(t)\right]$, as $t \in\left[t_{j}, t_{j+1}\right]$.

Notice that if $L$ and $U$ are both constant or linear, the barriers defined above do coincide with the original ones. Otherwise, it is easy to see that for any $n \geq n_{0}=$ $\left.\max \left(1, \frac{H}{D}\right)\right)$, where $D=\min _{t \in[0, T]}(U(t)-L(t))$ and $H=\max _{t, s \in[0, T]}\left(L^{\prime}(t)-U^{\prime}(s)\right)$, one has $\phi_{L, 1}^{j}(t) \leq \phi_{U, 1}^{j}(t)$ as $t \in\left[t_{j}, t_{j+1}\right)$ for any $j \leq n$, so that

$$
\begin{gathered}
{\left[\phi_{L, 1}^{j}(t), \phi_{U, 1}^{j}(t)\right] \subset\left[\phi_{L}^{j}(t), \phi_{U}^{j}(t)\right] \subset\left[\phi_{L, 2}^{j}(t), \phi_{U, 2}^{j}(t)\right]} \\
{\left[\phi_{L, 1}^{j}(t), \phi_{U, 1}^{j}(t)\right] \subset[L(t), U(t)] \subset\left[\phi_{L, 2}^{j}(t), \phi_{U, 2}^{j}(t)\right] .}
\end{gathered}
$$

From now on, we suppose $n \geq n_{0}$, so that (10) holds.

For any $(x, y) \in\left(L_{j}, U_{j}\right) \times\left(L_{j+1}, U_{j+1}\right)$, let us now define $p_{L, i, n}^{j}(x, y)$ and $p_{U, i, n}^{j}(x, y)$ as the hitting probability on $\phi_{L, i}^{j}$ and on $\phi_{U, i}^{j}$, respectively as $i \in\{1,2\}$. By recalling that $\hat{p}_{n}^{j}$ turns out to be the exit probability from the double barriers $\phi_{L}^{j}$ and $\phi_{U}^{j}$, from (10) it easily follows that

$$
\begin{aligned}
& \max \left(p_{L, 2, n}^{j}(x, y), p_{U, 2, n}^{j}(x, y)\right) \leq \tilde{p}_{n}^{j}(x, y) \leq p_{L, 1, n}^{j}(x, y)+p_{U, 1, n}^{j}(x, y) \\
& \max \left(p_{L, 2, n}^{j}(x, y), p_{U, 2, n}^{j}(x, y)\right) \leq p_{n}^{j}(x, y) \leq p_{L, 1, n}^{j}(x, y)+p_{U, 1, n}^{j}(x, y)
\end{aligned}
$$


and then

$$
\frac{\left|p_{n}^{j}(x, y)-\hat{p}_{n}^{j}(x, y)\right|}{\hat{p}_{n}^{j}(x, y)} \leq \frac{p_{L, 1, n}^{j}(x, y)+p_{U, 1, n}^{j}(x, y)-\max \left(p_{L, 2, n}^{j}(x, y), p_{U, 2, n}^{j}(x, y)\right)}{\max \left(p_{L, 2, n}^{j}(x, y), p_{U, 2, n}^{j}(x, y)\right)}
$$

Let us set

$$
\begin{aligned}
& \Gamma_{n}^{j}=\frac{p_{L, 1, n}^{j}(x, y)+p_{U, 1, n}^{j}(x, y)-p_{L, 2, n}^{j}(x, y)}{p_{L, 2, n}^{j}(x, y)} \\
& \Lambda_{n}^{j}=\frac{p_{L, 1, n}^{j}(x, y)+p_{U, 1, n}^{j}(x, y)-p_{U, 2, n}^{j}(x, y)}{p_{U, 2, n}^{j}(x, y)}
\end{aligned}
$$

so that

$$
\frac{\left|p_{n}^{j}(x, y)-\widehat{p}_{n}^{j}(x, y)\right|}{\widehat{p}_{n}^{j}(x, y)} \leq \min \left(\Gamma_{n}^{j}, \Lambda_{n}^{j}\right) .
$$

If $w^{j}=\left(U_{j}-x\right)\left(U_{j+1}-y\right)-\left(L_{j}-x\right)\left(L_{j+1}-y\right)$, straightforward computations allow to deduce that

$$
\begin{aligned}
\Gamma_{n}^{j} \leq \exp \left(-\frac{2 n}{\sigma^{2}} w^{j}+\frac{2 n}{\sigma^{2}}\left[\left(x-L_{j}\right)\left(L_{j+1}-L_{j}-\frac{1}{n} L^{\prime}\left(t_{2}^{*}\right)\right)+\left(U_{j}-x\right)\left(U_{j+1}-U_{j}-\frac{1}{n} U^{\prime}\left(t_{1}^{\circ}\right)\right)\right]\right) \\
\quad+\exp \left(\frac{2}{\sigma^{2}}\left(x-L_{j}\right)\left(L^{\prime}\left(t_{1}^{*}\right)-L^{\prime}\left(t_{2}^{*}\right)\right)\right)-1, \\
\Lambda_{n}^{j} \leq \exp \left(\frac{2 n}{\sigma^{2}} w^{j}+\frac{2 n}{\sigma^{2}}\left[\left(x-L_{j}\right)\left(L_{j}+\frac{1}{n} L^{\prime}\left(t_{1}^{*}\right)-L_{j+1}\right)+\left(U_{j}-x\right)\left(U_{j}+\frac{1}{n} U^{\prime}\left(t_{2}^{\circ}\right)-U_{j+1}\right)\right]\right) \\
+\exp \left(\frac{2}{\sigma^{2}}\left(U_{j}-x\right)\left(U^{\prime}\left(t_{2}^{\circ}\right)-U^{\prime}\left(t_{1}^{\circ}\right)\right)\right)-1 .
\end{aligned}
$$

Now, since $L$ and $U$ have Lipschitz continuous derivatives, with Lipschitz constant $K_{L}^{\prime}$ and $K_{U}^{\prime}$, respectively one has

$$
\begin{aligned}
& 0 \leq L_{j+1}-L_{j}-\frac{1}{n} L^{\prime}\left(t_{2}^{*}\right) \leq \frac{K_{L}^{\prime}}{n^{2}}, 0 \leq U_{j+1}-U_{j}-\frac{1}{n} U^{\prime}\left(t_{1}^{\circ}\right) \leq \frac{K_{U}^{\prime}}{n^{2}}, 0 \leq L^{\prime}\left(t_{1}^{*}\right)-L^{\prime}\left(t_{2}^{*}\right) \leq \frac{K_{L}^{\prime}}{n}, \\
& 0 \leq L_{j}+\frac{1}{n} L^{\prime}\left(t_{1}^{*}\right)-L_{j+1} \leq \frac{K_{L}^{\prime}}{n^{2}}, 0 \leq U_{j}+\frac{1}{n} U^{\prime}\left(t_{2}^{\circ}\right)-U_{j+1} \leq \frac{K_{U}^{\prime}}{n^{2}}, 0 \leq U^{\prime}\left(t_{2}^{\circ}\right)-L^{\prime}\left(t_{1}^{\circ}\right) \leq \frac{K_{U}^{\prime}}{n} .
\end{aligned}
$$

Thus, setting $K^{\prime}=\max \left(K_{U}^{\prime}, K_{L}^{\prime}\right)$, one obtains

$$
\Gamma_{n}^{j} \leq e^{-\frac{2 n}{\sigma^{2}} w^{j}+\frac{4 M K^{\prime}}{n \sigma^{2}}}+e^{\frac{2 M K^{\prime}}{n \sigma^{2}}}-1, \quad \Lambda_{n}^{j} \leq e^{\frac{2 n}{\sigma^{2}} w^{j}+\frac{4 M K K^{\prime}}{n \sigma^{2}}}+e^{\frac{2 M K^{\prime}}{n \sigma^{2}}}-1
$$

where $M=\max _{t \in[0, T]}(U(t)-L(t))$. Recalling that $e^{t}-1 \leq t e^{t}$ for any positive $t$, it holds

$$
\Gamma_{n}^{j} \leq e^{\frac{4 M K^{\prime}}{n \sigma^{2}}-\frac{2 n}{\sigma^{2}} w^{j}}+\frac{2 M K^{\prime}}{n \sigma^{2}} e^{\frac{2 M K^{\prime}}{n \sigma^{2}}}, \quad \Lambda_{n}^{j} \leq e^{\frac{4 M K^{\prime}}{n \sigma^{2}}+\frac{2 n}{\sigma^{2}} w^{j}}+\frac{2 M K^{\prime}}{n \sigma^{2}} e^{\frac{2 M K^{\prime}}{n \sigma^{2}}},
$$

so that

$$
\min \left(\Gamma_{n}^{j}, \Lambda_{n}^{j}\right) \leq e^{\frac{4 M K^{\prime}}{n \sigma^{2}}-\frac{2 n}{\sigma^{2}}\left|w^{j}\right|}+\frac{2 M K^{\prime}}{n \sigma^{2}} e^{\frac{4 M K^{\prime}}{n \sigma^{2}}}
$$

Therefore, if $\left|w^{j}\right| \geq \frac{\alpha \sigma^{2} \ln n}{2 n}$, it follows that

$$
\frac{\left|p_{n}^{j}(x, y)-\widehat{p}_{n}^{j}(x, y)\right|}{\widehat{p}_{n}^{j}(x, y)} \leq \min \left(\Gamma_{n}^{j}, \Lambda_{n}^{j}\right) \leq \frac{e^{\frac{\Delta M K^{\prime}}{n \sigma^{2}}}}{n^{\alpha}}+\frac{2 M K^{\prime}}{n \sigma^{2}} e^{\frac{2 M K^{\prime}}{n \sigma^{2}}}
$$


and the statement finally holds.

We are now ready to prove next result, which turns out to be the principal part of the proof of Theorem 2.3.

Proposition 3.3 Let $\alpha>1$ and $f$ be a positive function such that $\mathbb{E}\left[f\left(W_{T}\right)^{1+\epsilon}\right]<+\infty$ for some $\epsilon>0$. Then there exists a positive integer $N_{0}$ such that for any $n \geq N_{0}$,

$$
\int_{\prod_{j=0}^{n-1}\left(L_{j}, U_{j}\right)} f\left(x_{n}\right)\left|q_{n}(x)-\widehat{q}_{n}(x)\right| d \mu_{n}(x) \leq\left(\frac{c_{n}}{n}+\frac{d_{n}}{n^{\alpha}}\right) \int_{\prod_{j=0}^{n-1}\left(L_{j}, U_{j}\right)} f\left(x_{n}\right) q_{n}(x) d \mu_{n}(x)+\gamma_{n}
$$

where $\left\{\gamma_{n}\right\}_{n}$ is a sequence going to 0 faster than any power of $\frac{1}{n}$, as $n \rightarrow+\infty$.

Proof. Let us first set $A:=\prod_{j=0}^{n-1}\left(L_{j}, U_{j}\right)=\cup_{j=0}^{n-1} A_{j}$ where $A_{0}=\left\{x \in A:\left|w^{i}\left(x_{i}, x_{i+1}\right)\right|>\right.$ $\left.\frac{\alpha \sigma^{2} \ln n}{2 n} \forall i\right\}$ and, for $j \geq 1, A_{j}=\cup_{I \in \mathcal{I}_{j}} A_{j, I}$, being

$$
\mathcal{I}_{j}=\{I \subset\{1, \ldots, n-1\}: \# I=j\}
$$

and

$$
A_{j, I}=\left\{x \in A:\left|w^{i}\left(x_{i}, x_{i+1}\right)\right|>\frac{\alpha \sigma^{2} \ln n}{2 n} \forall i \notin I,\left|w^{i}\left(x_{i}, x_{i+1}\right)\right| \leq \frac{\alpha \sigma^{2} \ln n}{2 n} \forall i \in I\right\} .
$$

Let us fix now $\delta>0$, which we shall suitably choose later, and set

$$
C_{\delta}^{j}=\left(L_{j}, L_{j}+\delta\right) \times\left(U_{j+1}-\delta m_{j}, U_{j+1}\right) \cup\left(U_{j}-\delta, U_{j}\right) \times\left(L_{j+1}, L_{j+1}+\delta m_{j}\right)
$$

being $m_{j}=\frac{U_{i+1}-L_{j+1}}{U_{j}-L_{j}}$. Since we need that $C_{\delta}^{j} \subset\left(L_{j}, U_{j}\right) \times\left(L_{j+1}, U_{j+1}\right)$, and $C_{\delta}^{i}$ is the union of two non intersecting set, for the moment it is sufficient to require $\delta<\frac{D}{2}$, where $D=\min _{t \in[0, T]}(U(t)-L(t))$.

We can then set

$$
A_{j, I, 0}=A_{j, I} \cap\left\{x \in A: \forall i \in I\left(x_{i}, x_{i+1}\right) \notin C_{\delta}^{i}\right\}
$$

and

$$
A_{j, I, 1}=A_{j, I} \cap\left\{x \in A: \exists i \in I\left(x_{i}, x_{i+1}\right) \in C_{\delta}^{i}\right\}
$$

so that

$$
\begin{gathered}
\int_{A} f\left(x_{n}\right)\left|q_{n}(x)-\widehat{q}_{n}(x)\right| d \mu_{n}(x)=\int_{A_{0}} f\left(x_{n}\right)\left|q_{n}(x)-\widehat{q}_{n}(x)\right| d \mu_{n}(x) \\
+\int_{\cup_{j=1}^{n-1} \cup_{I \in I_{j}} A_{j, I, 0}} f\left(x_{n}\right)\left|q_{n}(x)-\widehat{q}_{n}(x)\right| d \mu_{n}(x)+\int_{\cup_{j=1}^{n-1} \cup_{I \in I_{j}} A_{j, I, 1}} f\left(x_{n}\right)\left|q_{n}(x)-\widehat{q}_{n}(x)\right| d \mu_{n}(x) \\
=\Gamma_{1}+\Gamma_{2}+\Gamma_{3} .
\end{gathered}
$$

We first study the term $\Gamma_{1}$. Suppose that $x \in A_{0}$, that is $\left|w^{i}\left(x_{i}, x_{i+1}\right)\right|>\frac{\alpha \sigma^{2} \ln n}{2 n}$ for any $i$, by Lemma 3.2, there exists $n_{0}$ such that for any $n \geq n_{0}$ one has

$$
\left|p^{i}\left(x_{i}, x_{i+1}\right)-\hat{p}^{i}\left(x_{i}, x_{i+1}\right)\right| \leq\left(\frac{c_{n}}{n}+\frac{d_{n}}{n^{\alpha}}\right) p^{i}\left(x_{i}, x_{i+1}\right)
$$


as $i=0,1, \ldots, n-1$. So, by applying Lemma 3.1, one obtains $\left|q_{n}(x)-\widehat{q}_{n}(x)\right| \leq\left(\frac{c_{n}}{n}+\right.$ $\left.\frac{d_{n}}{n^{a}}\right) q_{n}(x)$ and

$$
\Gamma_{1}=\int_{A_{0}} f\left(x_{n}\right)\left|q_{n}(x)-\widehat{q}_{n}(x)\right| d \mu_{n}(x) \leq\left(\frac{c_{n}}{n}+\frac{d_{n}}{n^{\alpha}}\right) \int_{A_{0}} f\left(x_{n}\right) q_{n}(x) d \mu_{n}(x) .
$$

Consider now $\Gamma_{2}=\sum_{j=1}^{n-1} \sum_{I \in \mathcal{I}_{j}} \int_{A_{j, I, 0}} f\left(x_{n}\right)\left|q_{n}(x)-\widehat{q}_{n}(x)\right| d \mu_{n}(x)$. Let us fix $j \in\{1, \ldots, n-$ $1\}$ and $I \in \mathcal{I}_{j}$ and take $x \in A_{j, I, 0}$. Then,

$$
\begin{gathered}
q_{n}(x)-\widehat{q}_{n}(x)=\left(1-\prod_{i \notin I}\left(1-p_{n}^{i}\left(x_{i}, x_{i+1}\right)\right)\right)-\left(1-\prod_{i \notin I}\left(1-\hat{p}_{n}^{i}\left(x_{i}, x_{i+1}\right)\right)\right) \\
+\prod_{i \notin I}\left(1-p_{n}^{i}\left(x_{i}, x_{i+1}\right)\right)\left(1-\prod_{i \in I}\left(1-p_{n}^{i}\left(x_{i}, x_{i+1}\right)\right)\right)-\prod_{i \notin I}\left(1-\hat{p}_{n}^{i}\left(x_{i}, x_{i+1}\right)\right)\left(1-\prod_{i \in I}\left(1-\hat{p}_{n}^{i}\left(x_{i}, x_{i+1}\right)\right)\right) .
\end{gathered}
$$

If $i \notin I$, Lemma 3.2 states that condition (*) holds, so that Lemma 3.1 can be applied, giving

$$
\left|\left(1-\prod_{i \notin I}\left(1-p_{n}^{i}\left(x_{i}, x_{i+1}\right)\right)\right)-\left(1-\prod_{i \notin I}\left(1-\hat{p}_{n}^{i}\left(x_{i}, x_{i+1}\right)\right)\right)\right| \leq\left(\frac{c_{n}}{n}+\frac{d_{n}}{n^{\alpha}}\right)\left(1-\prod_{i \notin I}\left(1-p_{n}^{i}\left(x_{i}, x_{i+1}\right)\right)\right) .
$$

Since $\left(1-\prod_{i \notin I}\left(1-p_{n}^{i}\left(x_{i}, x_{i \div 1}\right)\right)\right) \leq\left(1-\prod_{i=1}^{n-1}\left(1-p_{n}^{i}\left(x_{i}, x_{i+1}\right)\right)\right)=q_{n}(x)$, we can write

$$
\left|q_{n}(x)-\widehat{q}_{n}(x)\right| \leq\left(\frac{c_{n}}{n}+\frac{d_{n}}{n^{\alpha}}\right) q_{n}(x)+\left(1-\prod_{i \in I}\left(1-p_{n}^{i}\left(x_{i}, x_{i+1}\right)\right)\right)+\left(1-\prod_{i \in I}\left(1-\hat{p}_{n}^{i}\left(x_{i}, x_{i+1}\right)\right)\right) .
$$

In order to estimate the latter two quantities on the right hand side of $(* *)$, we need to use the probabilities $p_{L, 1, n}^{i}$ and $p_{L, 1, n}^{i}$ which have been used in Lemma 3.2. More precisely, we consider the constraints in (11), holding for any $n \geq n_{0}$ :

$$
\begin{gathered}
p_{n}^{i}\left(x_{i}, x_{i+1}\right) \leq p_{L, 1, n}^{i}\left(x_{i}, x_{i+1}\right)+p_{U, 1, n}^{i}\left(x_{i}, x_{i+1}\right) \\
=\exp \left(-\frac{2 n}{\sigma^{2}}\left(L_{i}-x_{i}\right)\left(L_{i}+\frac{1}{n} L^{\prime}\left(t_{1}^{*}\right)-x_{i+1}\right)\right)+\exp \left(-\frac{2 n}{\sigma^{2}}\left(U_{i}-x_{i}\right)\left(U_{i}+\frac{1}{n} U^{\prime}\left(t_{1}^{\circ}\right)-x_{i+1}\right)\right) \\
\leq \exp \left(-\frac{2 n}{\sigma^{2}}\left(L_{i}-x_{i}\right)\left(L_{i+1}-x_{i+1}\right)\right) \exp \left(\frac{2 n}{\sigma^{2}}\left(L_{i}+\frac{1}{n} L^{\prime}\left(t_{1}^{*}\right)-L_{i+1}\right)\left(x_{i}-L_{i}\right)\right) \\
\quad+\exp \left(-\frac{2 n}{\sigma^{2}}\left(U_{i}-x_{i}\right)\left(U_{i+1}-x_{i+1}\right)\right) \exp \left(\frac{2 n}{\sigma^{2}}\left(U_{i+1}-U_{i}+\frac{1}{n} U^{\prime}\left(t_{1}^{\circ}\right)\right)\left(U_{i}-x_{i}\right)\right) \\
\leq \mathrm{e}^{\frac{2 M K_{L}^{\prime}}{n \sigma^{2}}} \exp \left(-\frac{2 n}{\sigma^{2}}\left(L_{i}-x_{i}\right)\left(L_{i+1}-x_{i+1}\right)\right)+\mathrm{e}^{\frac{2 M K_{u}^{\prime}}{n \sigma^{2}}} \exp \left(-\frac{2 n}{\sigma^{2}}\left(U_{i}-x_{i}\right)\left(U_{i+1}-x_{i+1}\right)\right) \\
\leq \mathrm{e}^{\frac{2 M K^{\prime}}{n \sigma^{2}}}\left[\exp \left(-\frac{2 n}{\sigma^{2}}\left(L_{i}-x_{i}\right)\left(L_{i+1}-x_{i+1}\right)\right)+\exp \left(-\frac{2 n}{\sigma^{2}}\left(U_{i}-x_{i}\right)\left(U_{i+1}-x_{i+1}\right)\right)\right] .
\end{gathered}
$$

Similarly, we can state, for any $n \geq n_{0}$,

$\hat{p}_{n}^{i}\left(x_{i}, x_{i+1}\right) \leq \mathrm{e}^{\frac{2 M K^{\prime}}{n \sigma^{2}}}\left[\exp \left(-\frac{2 n}{\sigma^{2}}\left(L_{i}-x_{i}\right)\left(L_{i+1}-x_{i+1}\right)\right)+\exp \left(-\frac{2 n}{\sigma^{2}}\left(U_{i}-x_{i}\right)\left(U_{i+1}-x_{i+1}\right)\right)\right]$.

Now, if $i \in I$ then $\left(x_{i}, x_{i+1}\right) \notin C_{\delta}^{i}$, so

$$
\left(L_{i}-x_{i}\right)\left(L_{i+1}-x_{i+1}\right) \geq\left(\delta-\frac{\alpha \sigma^{2} \ln n}{2 n}\right)\left(\delta m_{i}-\frac{\alpha \sigma^{2} \ln n}{2 n}\right)
$$




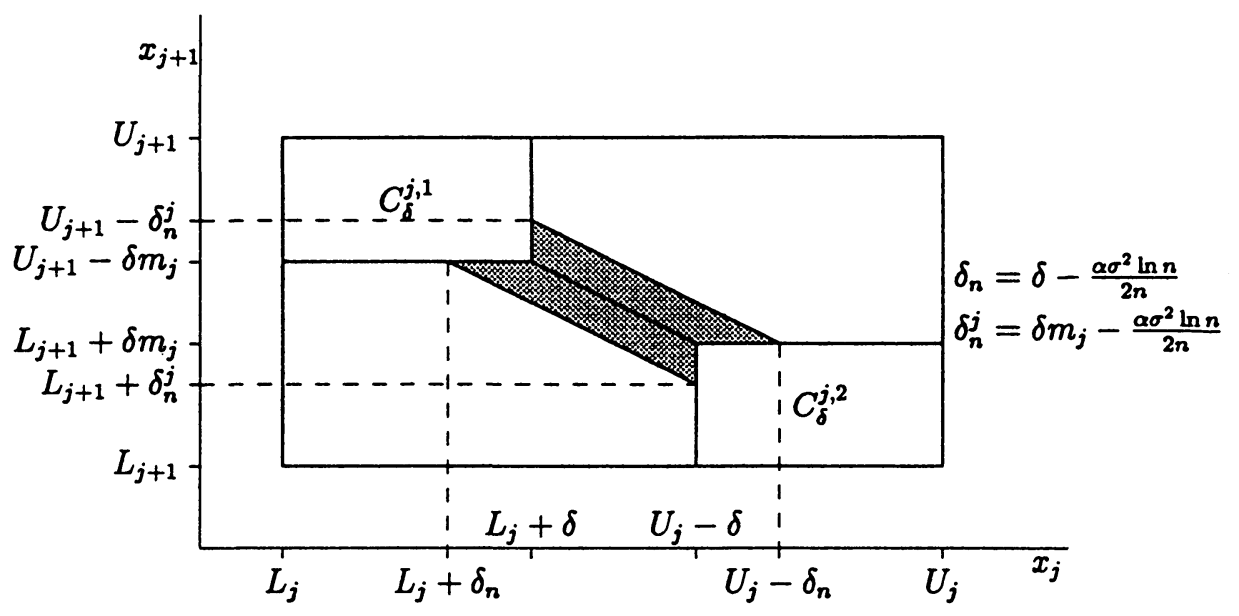

Figure 1 For $n$ large enough, the shadowed region shows the set of points $\left(x_{j}, x_{j+1}\right) \notin C_{\delta}^{j}=$ $C_{\delta}^{j, 1} \cup C_{\delta}^{j, 2}$ such that $w^{j}\left(x_{j}, x_{j+1}\right) \leq \frac{\alpha \sigma^{2} \ln n}{2 n}$.

and

$$
\left(U_{i}-x_{i}\right)\left(U_{i+1}-x_{i+1}\right) \geq\left(\delta-\frac{\alpha \sigma^{2} \ln n}{2 n}\right)\left(\delta m_{i}-\frac{\alpha \sigma^{2} \ln n}{2 n}\right)
$$

hold, as it is easily seen from Figure 1 , and are obviously of interest whenever $\delta$ is chosen in order to make the right hand sides positive.

Thus, let $\delta<\frac{D}{2}$ and $n_{1}$ such that $\frac{\delta}{2}>\frac{M}{D} \frac{\alpha \sigma^{2} \ln n_{1}}{n_{1}}$ (where the quantity $\frac{M}{D}$ comes from an upper bound for $\left.m_{j}\right)$, so that for any $n \geq \max \left(n_{0}, n_{1}\right)$ one has

$$
\left(L_{i}-x_{i}\right)\left(L_{i+1}-x_{i+1}\right) \geq \frac{\delta^{2}}{4} \quad \text { and } \quad\left(U_{i}-x_{i}\right)\left(U_{i+1}-x_{i+1}\right) \geq \frac{\delta^{2}}{4} .
$$

For such values of $n$, if $i \in I$, we can then give the following estimates

$$
p_{n}^{i}\left(x_{i}, x_{i+1}\right) \leq 2 e^{\frac{2 M K^{\prime}}{n \sigma^{2}}-\frac{n \delta^{2}}{2 \sigma^{2}}} \quad \text { and } \quad \hat{p}_{n}^{i}\left(x_{i}, x_{i+1}\right) \leq 2 e^{\frac{2 M K^{\prime}}{n \sigma^{2}}-\frac{n \delta^{2}}{2 \sigma^{2}}}
$$

For $n$ large enough, $2 e^{\frac{2 . M K^{\prime}}{n \sigma^{2}}-\frac{n \delta^{2}}{2 \sigma^{2}}}<1$ (indeed, it is sufficient to take $n \geq n_{2}$, where $n_{2}$ is such that $\left.n_{2}^{2} \delta^{2}-n_{2} \sigma^{2}-4 M K>0\right)$, so that

$$
1-\prod_{i \in I}\left(1-p_{n}^{i}\left(x_{i}, x_{i \div 1}\right)\right) \leq 1-\left(1-2 e^{\frac{2 M K^{\prime}}{n \sigma^{2}}-\frac{n \delta^{2}}{2 \sigma^{2}}}\right)^{j} \leq 1-\left(1-2 e^{\frac{2 M K^{\prime}}{n \sigma^{2}}-\frac{n \delta^{2}}{2 \sigma^{2}}}\right)^{n}
$$

and similarly

$$
1-\prod_{i \in I}\left(1-\hat{p}_{n}^{i}\left(x_{i}, x_{i+1}\right)\right) \leq 1-\left(1-2 a_{n} \mathrm{e}^{-\frac{n \delta^{2}}{2 \sigma^{2}}}\right)^{n} .
$$

Therefore, for $x \in A_{j, I, 0}$ and $n \geq \max \left(n_{0}, n_{1}, n_{2}\right)$, from (**) it follows that

$$
\left|q_{n}(x)-\widehat{q}_{n}(x)\right| \leq\left(\frac{c_{n}}{n}+\frac{d_{n}}{n^{\alpha}}\right) q_{n}(x)+\gamma_{n}^{1}
$$

where

$$
\gamma_{n}^{1}=2\left(1-\left(1-2 e^{\frac{2 M I K^{\prime}}{n \sigma^{2}}-\frac{n \delta^{2}}{2 \sigma^{2}}}\right)^{n}\right)
$$


and finally

$$
\begin{gathered}
\Gamma_{2}=\int_{\cup_{j=1}^{n-1} \cup_{I \in \mathcal{I}_{j}} A_{j, I, 0}} f\left(x_{n}\right)\left|q_{n}(x)-\widehat{q}_{n}(x)\right| d \mu_{n}(x) \\
\leq\left(\frac{c_{n}}{n}+\frac{d_{n}}{n^{\alpha}}\right) \int_{\bigcup_{j=1}^{n-1} \cup_{I \in \mathcal{I}_{j}} A_{j, I, 0}} f\left(x_{n}\right) q_{n}(x) d \mu_{n}(x)+\gamma_{n}^{1} \cdot \mathbb{E}\left[f\left(W_{T}\right)\right] .
\end{gathered}
$$

For $\Gamma_{3}$, notice that

$$
\cup_{j=1}^{n-1} \cup_{I \in \mathcal{I}_{j}} A_{j, I, 1} \subset \cup_{k=0}^{n-1}\left\{x \in A ;\left(x_{k}, x_{k+1}\right) \in C_{\delta}^{k}\right\}
$$

so that

$$
\begin{gathered}
\Gamma_{3}=\int_{\cup_{j=1}^{n-1} \cup_{I \in x_{j}} A_{j, I, 1}} f\left(x_{n}\right) q_{n}(x) d \mu_{n}(x) \leq \sum_{k=0}^{n-1} \int_{\left\{x \in A ;\left(x_{k}, x_{k+1}\right) \in C_{\delta}^{k}\right\}} f\left(x_{n}\right) d \mu_{n}(x) \\
\leq\left(\sum_{k=0}^{n-1} \int_{\left\{x \in A ;\left(x_{k}, x_{k+1}\right) \in C_{\delta}^{k}\right\}} f\left(x_{n}\right)^{1+\epsilon} d \mu_{n}(x)\right)^{\frac{1}{1+e}}\left(\sum_{k=0}^{n-1} \int_{\left\{x \in A ;\left(x_{k}, x_{k+1}\right) \in C_{\delta}^{k}\right\}} d \mu_{n}(x)\right)^{\frac{e}{1+\epsilon}} \\
\leq\left(\mathbb{E}\left[f\left(W_{T}\right)^{1+\epsilon}\right]\right)^{\frac{1}{1+e}} \cdot\left(\sum_{k=0}^{n-1} \int_{C_{\delta}^{k}} P\left(W_{t_{k}} \in d x, W_{t_{k+1}} \in d y\right)\right)^{\frac{e}{1+\epsilon}} \\
\left(\mathbb{E}\left[f\left(W_{T}\right)^{1+\epsilon}\right]\right)^{\frac{1}{1+\epsilon}} \cdot\left(\sum_{k=0}^{n-1} \int_{C_{\delta}^{k}} P\left(W_{t_{k}} \in d x\right) \sqrt{\frac{n}{2 \pi \sigma^{2}}} e^{-\frac{n}{2 \sigma^{2}}(y-x)^{2}} d y\right)^{\frac{e}{1+e}}
\end{gathered}
$$

Now, if $(x, y) \in C_{\delta}^{k}$ then $\left\{\begin{array}{l}L_{k}<x<L_{k}+\delta \\ U_{k+1}-\delta m_{k}<y<U_{k+1}\end{array}\right.$ or $\left\{\begin{array}{l}U_{k}-\delta<x<U_{k} \\ L_{k+1}<y<L_{k+1}+\delta m_{k}\end{array}\right.$, so that

$$
\begin{aligned}
& y-x \geq U_{k+1}-L_{k}-\delta\left(1+m_{k}\right) \\
& x-y \geq U_{k}-L_{k+1}-\delta\left(1+m_{k}\right) .
\end{aligned}
$$

Since $U_{k+1}-L_{k} \geq D-\frac{K_{U}}{n}, L_{k+1}-U_{k} \geq D-\frac{K_{L}}{n}, K_{U}$ and $K_{L}$ being the Lipschitz constant of $L$ and $U$, respectively and $m_{k} \leq \frac{M}{D}$, setting $K=\max \left(K_{L}, K_{U}\right)$ we can write

$$
|y-x| \geq D-\frac{K}{n}-\delta\left(1+\frac{M}{D}\right)
$$

whenever $(x, y) \in C_{\delta}^{k}$, for any $k$ (recall that $D-\frac{k}{n}>0$ if $n>\frac{K}{D}$ ). Thus, choosing

$$
0<\delta \leq \frac{1}{2}\left(\frac{D-K \frac{1}{n}}{1+\frac{M}{D}}\right)
$$

then for any $(x, y) \in C_{\delta}^{k}$ and for any $k$ one obtains $|y-x| \geq \frac{1}{2}\left(D-\frac{K}{n}\right)$ and

$$
\int_{C_{\delta}^{k}} P\left(B_{t_{k}} \in d x\right) \sqrt{\frac{n}{2 \pi \sigma^{2}}} e^{-\frac{n}{2 \sigma^{2}}(y-x)^{2}} d y \leq \sqrt{\frac{n}{2 \pi \sigma^{2}}} e^{-\frac{n}{8 \sigma^{2}}\left(D-\frac{K}{n}\right)^{2}} .
$$

Therefore

$$
\begin{aligned}
& \Gamma_{3} \leq\left(\mathbb{E}\left[f\left(W_{T}\right)^{1+\epsilon}\right]\right)^{\frac{1}{1+e}} \cdot\left(\sum_{k=0}^{n-1} \int_{C_{\delta}^{k}} P\left(B_{t_{k}} \in d x\right) \sqrt{\frac{n}{2 \pi \sigma^{2}}} e^{-\frac{n}{2 \sigma^{2}}(y-x)^{2}} d y\right)^{\frac{e}{1-\epsilon}} \\
& \leq\left(\mathbb{E}\left[f\left(W_{T}\right)^{1+\epsilon}\right]\right)^{\frac{1}{1+\epsilon}} \cdot\left(\frac{n \sqrt{n}}{\sqrt{2 \pi \sigma^{2}}} e^{-\frac{n}{8 \sigma^{2}}\left(D-\frac{K}{n}\right)^{2}}\right)^{\frac{e}{1-\epsilon}}=:\left(\mathbb{E}\left[f\left(W_{T}\right)^{1+\epsilon}\right]\right)^{\frac{1}{1+\epsilon}} \cdot \gamma_{n}^{2}
\end{aligned}
$$


Finally, the statement holds with $\gamma_{n}=\mathbb{E}\left[f\left(W_{T}\right)\right] \gamma_{n}^{1}+\left(\mathbb{E}\left[f\left(W_{T}\right)^{1+\epsilon}\right)^{\frac{1}{1+e}} \gamma_{n}^{2}\right.$, which goes to 0 as $n \rightarrow \infty$ exponentially fast.

Proof of Theorem 2.3. Let us set $A_{n}=\prod_{j=0}^{n-1}\left(L_{j}, U_{j}\right)$. First of all, notice that

$$
\mathbb{E}\left[f\left(W_{T}\right) \mathbb{I}_{\{\tau>t\}}\right]=\int_{A_{n}} f\left(x_{n}\right) \cdot\left(1-q_{n}(x)\right) d \mu_{n}(x)
$$

and

$$
\mathbb{E}\left[f\left(W_{T}\right) \mathbb{1}_{\left\{\tau_{n}^{c}>t\right\}}\right]=\int_{A_{n}} f\left(x_{n}\right) \cdot\left(1-\widehat{q}_{n}(x)\right) d \mu_{n}(x)
$$

By using Proposition 3.3, we have

$$
\mathcal{E}_{n}^{c}(f) \leq \int_{A_{n}} f\left(x_{n}\right) \cdot\left|\widehat{q}_{n}(x)-q_{n}(x)\right| d \mu_{n}(x) \leq\left(\frac{c_{n}}{n}+\frac{d_{n}}{n^{\alpha}}\right) \int_{A_{n}} f\left(x_{n}\right) \cdot q_{n}(x) d \mu_{n}(x)+\gamma_{n}
$$

It is now sufficient to show that $\mathcal{E}_{n}^{d}=\int_{A_{n}} f\left(x_{n}\right) \cdot q_{n}(x) d \mu_{n}(x)$. Indeed, since $\tau_{n}^{d} \geq \tau$, one can write

$$
\mathcal{E}_{n}^{d}=\left|\mathbb{E}\left[f\left(W_{T}\right)\left(\mathbb{I}_{\{\tau>T\}}-\mathbb{I}_{\left\{\tau_{n}^{d}>T\right\}}\right)\right]\right|=\mathbb{E}\left[f\left(W_{T}\right) \mathbb{1}_{\left\{\tau \leq T, \tau_{n}^{d}>T\right\}}\right]=\int_{A_{n}} f\left(x_{n}\right) \cdot q_{n}(x) d \mu_{n}(x) .
$$

Let us show some further consequences of the estimates given by Proposition 3.3.

Remark 3.4 If $f$ is not positive we cannot directly compare $\mathcal{E}_{n}^{c}(f)$ and $\mathcal{E}_{n}^{d}(f)$, but if $f$ is such that $\mathbb{E}\left[\left|f\left(W_{T}\right)\right|^{1+\epsilon}\right] \leq+\infty$ for some $\epsilon>0$ then by simple calculations we have

$$
\mathcal{E}_{n}^{c}(f) \leq \mathcal{E}_{n}^{d}(|f|) \varepsilon_{n}+\bar{\gamma}_{n}
$$

where $\varepsilon_{n}=\frac{c_{n}}{n}+\frac{d_{n}}{n^{a}}$, with $c_{n}$ and $d_{n}$ are given in Proposition 3.3, and $\bar{\gamma}_{n}$ is a sequence going to 0 as $n \rightarrow \infty$ faster than any power of $1 / n$.

Remark 3.5 In a financial framework, it is of interest also to compute quantities like

$$
\mathbb{E}\left[f\left(W_{T}\right) \mathbb{I}_{\{\tau>T\}}+R e^{-r \tau} \mathbb{I}_{\{\tau \leq T\}}\right]
$$

where $R$ is a positive constant called rebate. Indeed, if one thinks to knock-out barrier options, with payoff given through $f$, then $R$ stands for a quantity, typically quite small, that the option seller gives to the buyer in the event that the underlying asset price hits on the barriers (see e.g. [3]). Now, a simple application of Proposition 3.3 allows to control the error arising from the application of the continuous scheme for the pricing of barrier options with rebate. Let us stress the dependence of the error on both $f$ and $R$, that is

$$
\begin{aligned}
& \mathcal{E}_{n}^{c}(f, R)=\left|\mathbb{E}\left[f\left(W_{T}\right) \mathbb{1}_{\{\tau>T\}}+R e^{-r \tau} \mathbb{I}_{\{r \leq T\}}\right]-\mathbb{E}\left[f\left(W_{T}\right) \mathbb{1}_{\left\{\tau_{n}^{c}>T\right\}}+R e^{-r \tau_{n}^{c}} \mathbb{I}_{\left\{\tau_{n}^{c} \leq T\right\}}\right]\right| \\
& \mathcal{E}_{n}^{d}(f, R)=\left|\mathbb{E}\left[f\left(W_{T}\right) \mathbb{1}_{\{r>T\}}+R e^{-r \tau} \mathbb{I}_{\{\tau \leq T\}}\right]-\mathbb{E}\left[f\left(W_{T}\right) \mathbb{1}_{\left\{r_{n}^{d}>T\right\}}+R e^{-r \tau_{n}^{d}} \mathbb{I}_{\left\{\tau_{n}^{d} \leq T\right\}}\right]\right| .
\end{aligned}
$$

Then, it easily follows that

$$
\mathcal{E}_{n}^{c}(f, R) \leq\left(\mathcal{E}_{n}^{d}(f, 0)+\mathcal{E}_{n}^{d}(0, R)\right) \varepsilon_{n}+\bar{\gamma}_{n}
$$

where $\varepsilon_{n}=\frac{c_{n}}{n}+\frac{d_{n}}{n^{\alpha}}$, with $c_{n}$ and $d_{n}$ are given in Proposition 3.3 , and $\bar{\gamma}_{n}$ is a sequence going to 0 as $n \rightarrow \infty$ faster than any power of $1 / n$. Thus, in particular, $\lim _{n \rightarrow \infty} \mathcal{E}_{n}^{c}(f, R) / n=0$. This is the content of the next 
Proposition 3.6 Under the hypothesis of Theorem 2.3 and for any positive constant $R$, then (13) holds.

Proof. First of all, for any positive random variable $X$ we can write

$$
\mathbb{E}\left[e^{-r X} \mathbb{I}_{\{X \leq T\}}\right]=\int_{0}^{\infty} P\left(e^{-r X} \mathbb{1}_{\{X \leq T\}} \geq s\right) d s=\int_{0}^{1} P\left(X \leq T_{s}\right) d s
$$

with $T_{s}=T$ if $0<s<e^{-r T}$ and $T_{s}=-\frac{1}{r} \ln s$ if $e^{-r T} \leq s<1$. Thus,

$$
\mathcal{E}_{n}^{c}(f, R) \leq \mathcal{E}_{n}^{c}(f, 0)+R \int_{0}^{1}\left|P\left(\tau \leq T_{s}\right)-P\left(\tau_{n}^{c} \leq T_{s}\right)\right| d s
$$

Denoting by $q_{n}^{s}$ and $\widehat{q}_{n}^{s}$ the previously defined conditional exit probability and its approximation with $T$ replaced by $T_{s}$, one has

$$
\int_{0}^{1}\left|P\left(\tau \leq T_{s}\right)-P\left(\tau_{n}^{c} \leq T_{s}\right)\right| d s=\int_{0}^{1} \int_{\prod_{j=0}^{n-1}\left(L_{j}, U_{j}\right)}\left|q_{n}^{s}(x)-\widehat{q}_{n}^{s}(x)\right| \mu_{n}(d x) d s
$$

and by Proposition 3.3 (with $f \equiv 1$ ),

$$
\int_{0}^{1}\left|P\left(\tau \leq T_{s}\right)-P\left(\tau_{n}^{c} \leq T_{s}\right)\right| d s \leq \int_{0}^{1}\left[\left(\frac{c_{n}^{s}}{n}+\frac{d_{n}^{s}}{n^{\alpha}}\right) \int_{\prod_{j=0}^{n-1}\left(L_{j}, U_{j}\right)} q_{n}^{s}(x) d \mu_{n}(x)+\gamma_{n}^{s}\right] d s
$$

where $c_{n}^{s}, d_{n}^{s}$ and $\gamma_{n}^{s}$ are given in Proposition 3.3 with $T$ replaces by $T_{s}$. Now, since $T_{s} \leq T$ for any $s \in(0,1)$, it easily follows that $c_{n}^{s} \leq c_{n}$ and $d_{n}^{s} \leq d_{n}$, so that

$$
\begin{aligned}
& \int_{0}^{1}\left|P\left(\tau \leq T_{s}\right)-P\left(\tau_{n}^{c} \leq T_{s}\right)\right| d s \leq\left(\frac{c_{n}}{n}+\frac{d_{n}}{n^{\alpha}}\right) \int_{0}^{1} \int_{\prod_{j=0}^{n-1}\left(L_{j}, U_{j}\right)} q_{n}^{s}(x) d \mu_{n}(x) d s+\int_{0}^{1} \gamma_{n}^{s} d s \\
= & \left(\frac{c_{n}}{n}+\frac{d_{n}}{n^{\alpha}}\right) \int_{0}^{1}\left(P\left(\tau \leq T_{s}\right)-P\left(\tau_{n}^{d} \leq T_{s}\right)\right) d s+\int_{0}^{1} \gamma_{n}^{s} d s=\left(\frac{c_{n}}{n}+\frac{d_{n}}{n^{\alpha}}\right) \mathcal{E}_{n}^{d}(0,1)+\int_{0}^{1} \gamma_{n}^{s} d s .
\end{aligned}
$$

We can finally state that

$$
\mathcal{E}_{n}^{c}(f, R) \leq\left(\frac{c_{n}}{n}+\frac{d_{n}}{n^{\alpha}}\right)\left(\mathcal{E}_{n}^{d}(f, 0)+R \mathcal{E}_{n}^{d}(0,1)\right)+\gamma_{n}+\int_{0}^{1} \gamma_{n}^{s} d s
$$

and (13) holds.

\section{References}

[1] Baldi, P. (1995) Exact asymptotics for the probability of exit from a domain and applications to simulation. Annals of Probability, 23, 1644-1670.

[2] Baldi P., Caramellino L., Iovino M.G. (1999) Pricing general barrier options: a numerical approach using Sharp Large Deviations. Mathematical Finance, 9, 293-321. 
[3] Baldi P., Caramellino L., Iovino M.G. (1999) Pricing complex barrier options with general features using sharp large deviation estimates. Monte Carlo and Quasi-Monte Carlo Methods 1998, H. Niederreiter-J. Spanier (Eds.), Springer, 149-162.

[4] Boyle, P.P. and Tian, Y. (1997) Pricing lookback and barrier options under the CEV process. Journal of Financial and Quatitative Analysis, 34, 241-264.

[5] Broadie, M., Glasserman, P. and Kou, S. (1997) A Continuity Correction for Discrete Barrier Options. Mathematical Finance, 7, 325-349.

[6] Fleming W.H., James, M.R. (1992) Asymptotic series and exit time probability. Annals of Probability, 20, 1369-1384.

[7] Geman, H. and Yor, M. (1996) Pricing and Hedging Double-Barrier Options: a Probabilistic Approach. Mathematical Finance, 6, 365-378.

[8] Gobet, E. (2000) Weak approximations of killed diffusion using Euler schemes. Stochastic Processes and Their Applications, 87, 167-197.

[9] Kunitomo, N. and Ikeda, M. (1992) Pricing Options with Curved Boundaries. Mathematical Finance, 2, 275-297.

[10] Revuz, D., Yor, M. (1994) Continuous martingales and Brownian motion. 2nd edition. Springer, Berlin.

[11] Siegmund, D., and Yuh, Y.S. (1982) Brownian approximations for first passage probabilities. Z. Wahrscheinlichkeitsiheorie verw. Gebiete, 59, 238-248. 